\title{
The Introduction and the Application of Technological Innovations as Administrative Efficiency Factor in Education
}

\author{
Belias D. \\ Department of Physical Education and Sport Science, University of Thessaly, Trikala, Greece
}

Velissariou $\mathrm{E}$.

Dept. of Business Administration, Technological Educational Institute of Thessaly Greece

Kyriakou D.

Dept of Economic Sciences, Aristotle University of Thessaloniki, Thessaloniki, Greece

Koustelios a.

Department of Physical Education and Sport Science, University of Thessaly, Trikala, Greece

Sdrolias L.

Dept. of Business Administration, Technological Educational Institute of Thessaly Greece

Varsanis $\mathrm{K}$.

Dept. of Business Administration, Technological Educational Institute of Western Macedonia, Kozani, Greece.

\begin{abstract}
Innovation is a rapidly developed issue, keeping all the aforementioned alerted. Considering this picture, the key issue of this thesis is to clarify the concept innovation as administrative efficiency factor in relation with education using the relevant literature. The existing literature indicates that educational structure and practices are in the center of great reforms. These reforms associated with New Public Management. NPM is a process that involves interaction between managers and markets. It is a set of cost-cutting and management concepts from the private sector including downsizing, entrepreneurialism, enterprise operations, quality management, customer service etc. According to that concept, school managers are trying to create a smaller, more responsive, more entrepreneurial and more effective public sector. Technological innovation has a key role on this and surely it can be the cornerstone of every change which may occur on this field of public administration.
\end{abstract}

Keywords: technological innovations, school management, innovation, performance, efficiency

\section{Introduction}

As society struggles with fast changing circumstances, the cutting-edge in public governance becomes a high-aiming target. Public administration is at a stage where considerable pressure for change exists. Innovation in governance seems to repeat a historical pattern. It begins with administrative reforms to enhance the performance of public authorities, moves to changes in public policy, then involves the more difficult task of wider institutional reform and finally requires institutional innovation to complete the cycle of modernization (Caiden \& Puniha, 2011).

The UK Department of Business, Innovations and Skills give a typical definition of Public Sector Innovation (PSI): "Innovation is the process of identifying, testing implementing and spreading ideas that add value". Governments play an important role in establishing the conditions that will enable a knowledge- and innovation-driven economy to prosper. The withdrawal of administrative burdens, the subsidizing of specific research and developed projects and the launching of creative partnerships with businesses from the private sector are few of the government challenges. In addition, the deal with societal challenges, like education quality and crime fighting are also vital. 
The extended concern on how to utilize and achieve these concepts affects politicians, public servants and those whose seek to shape public opinion. Moreover innovation is a rapidly developed issue, keeping all the aforementioned alerted. Considering this picture, the key issue of this thesis is to clarify the concept innovation as administrative efficiency factor in relation with education using the relevant literature.

\section{Methodology}

This is a paper which is a clear literature review based on secondary data. Secondary Data is often includes surveys and researches that may have some relation with the research scopes but they do not always give the answers that the research is looking for. Nevertheless, they can help the author to gain knowledge and make comparisons with her research results.

This paper will rely on the presentation of the related literature review. Hence it will reproduce all of the related papers and research so to make up this paper.

\section{Literature Review}

\section{1. Educational institutions as organizations}

The term <organization> in the scientific management field comes with double meaning, which also consists of the definition:

a) as an entity (school, hospital or ministry)

b) as a basic managerial function (Katsaros,2008) The organizations are systems with coordinated activities, acts and forces that are brought by two or more people, who through communication they aim towards achieving one or more common goals. (Pavlopoulos,1983)

The "organization" -kindergarten, school, university, college or the educational system itself expects from the manager to do three things:

- to use the available resources in order to achieve his goals

- to maintain and develop the resources

- to be efficient The role of manager is different from the teacher's one, while the teacher is only responsible for the intellectual well being of the students, the manager on the other hand has to be the "glue" inside the organization connecting the different parts, facilitating the learning process and taking care of the financing and bureaucratic procedures.

\section{2. Educational Management and Leadership}

From the numerous researches that tried to give a definition in leadership by defining the concept, is noted its importance. Leadership is a process designed to influence the actions of a person or a group, in its effort to achieve the objectives of a company when the prevailing conditions are given. (Hersey \& Blanchard, 1969) Analyzing the definition of leadership it is obvious that the leader, the subordinates and other variables constitute the process of leadership (Mpourantas, 2005). Between leadership and management as well as between manager and leader there are differences (Foot \& Hook, 2008).

The necessity to change the behavior and the attitude of the people, when the prevailing conditions favor it, even to change the way that an organization operates or when an organization is trying to improve the working conditions as well as to implement an innovation, then we are referring to leadership. Leadership with the help of communication, aims to influence the behavior and the activities of the subordinates in order to achieve the goals. On the other hand, the use of bodies, sources of information and human resources in order to accomplish the aims of an organization, then we are talking about management. Managers form people's behavior through the official authority they possess. On the other hand the leader, with no official authority, demonstrated and recognized by a group of people, creates clear and perceptible values which are incorporated into the organization's strategy. Management deals with the non-human resources while leadership focuses on the human ones. Many efficient managers with the passage of the time become efficient leaders (Buchanan \& Huczynski, 2004).

Zileznik refers to four areas in order to define the differences between leaders and managers: their goals, their work, the image they have of themselves and the relations with others. Managers set goals that occur from the organization's needs 
and are objective, while leaders express their own vision, having personal perception about the goals. General, managers choose the most satisfying solution in order to achieve the organization's goals, the relationship with the subordinates is based on the power they have through the authority they possess and they seek stability inside the organization. Leaders are looking for innovative ways to solve a problem, develop emotional relationships with the subordinates, inspiring them and they don't take anything for granted, being always in search (Zileznik, 1977).

\section{3. Introduction to Educational Management}

Many researchers involved in the area of management and came up with a variety of definitions. The management is defined as the process by which the elements of a group are integrated, coordinated and utilized so as to efficiently achieve organizational objectives. (Carlisle, 1982) Management is defined as an operational process that includes five individual acts: programming, organization, administration, coordination and control (Saitis, 2008).

Saitis (1994) defines management as a rational combination of various activities that are included into a collaborative effort, inside an organization which is designed to serve specific purposes. Education is an area of management that shows both similarities with other areas and quite a few differences that require adaptation and new approaches. Specifying the definitions mentioned above at the school level the following findings are noted. The management function at school level, serves the purpose as mentioned at the law 1566/85 (article 1) that is defined as "purpose of the primary and secondary education is to contribute to the overall, harmonious and balanced development of the intellectual and psychosomatic abilities of the students" creating the conditions that will maximize the performance of the teaching and supportive staff, the local society and the parents. Implementing the definition of management in the field of education we could rephrase it as : a system of action that is based on the rational use of available resources - human and material- in order to fulfill the objectives that are set by the various types of the educational institutions (Saitis, 2000, p. 24), Indisputably the educational management is not only responsible for the implementation of the laws but also for the upgrade of the quality of the educational procedure that takes place at the school units (Saitis, 2008).

\section{4. Specifying innovation in the public administration}

In general terms public governance refers to a pattern of rules applying in the public sector. It conveys the administrative and process-oriented elements of governing. There are five separate categories that constitute the public governance (Kapucu, 2010):

\section{- Administrative governance concerns about public administration}

- Public policy governance shows the cooperation between networks and political elites

- Socio-political governance indicates institutional relationships in society

- Contract governance is a collection of policies controlling contracting-in and outsourcing practices

- Network governance analyzes the cooperation between government and nongovernmental organizations in order to promote communal interest.

More commonly public governance occurs through Networks (that engage public and private collaborations), Market mechanisms (where competition under government regulation allocate resources) and through methods that involve governments and the state bureaucracy. Innovation in public governance is an extended set of linked activities, a mechanism which is implemented to solve problems or to gain better governance outcomes. The increasing need for productivity and efficiency in the public sector gives innovation a leading part in all administrative activities engaged by government. It depicts the art of doing things in a better way than before.

Innovations diversify to incremental (those who evolve a current service or product) and to radical (those who launch something entirely new). Consider a product or service. Incremental innovation is to make the product better, simpler etc. Radical innovation is to launch a completely new product or service.

In addition, depending on who has initiated the process leading to behavioral changes, innovations are divided into "topdown" and "bottom- up". The first give priority at the top levels of management, meaning management or organizations or institutions higher up in the hierarchy. In the "bottom-up" process public employees, civil servants and mid-level policy 
makers are the groups that start the initiative. We make a more detailed approach on the following section "the diffusion process".

Finally, whether the innovation process has been initiated to solve a specific problem or in order to make already existing products, services or procedures more efficient it can be divided to needs- led and efficiency-led innovations. It has been shown that needs-led innovation is rarely driven by a major crisis but rather, mostly by internal problems (budget constraints, problems meeting the objective, etc.) which led to dynamic incremental innovation processes. Top-down innovations are probably less frequent than bottom-up innovation though more radical; they seem to be more driven by changes in the agencies' organization or pressure from the civil society (lobbying) than from legislative or electoral processes (Thenint, LL\&A 2010).

Innovations can take several forms:

- Product innovation- developments of products or services that an organization offers,

- Administrative innovation- the use of a new policy instrument, which may be a result of policy change,

- Process innovation- changes in the ways products/services created and delivered,

- System innovation- a new system or a fundamental change of an existing system, for example the establishment of new organizations or new patterns of co-operation and interaction,

- Paradigm innovation- shifts in the underlying mental models which frame what the organization does,

- Conceptual innovation- a change in the outlook of actors (such changes are accompanied by the use of new concepts),

- Radical change of rationality-meaning that the worldview or the mental matrix of the employees of an organization is shifting Rarity and significance can be seen as a precondition for innovation meaning that innovation is a relative phenomenon (Anttiroiko, Bailey \& Valkama, 2011).

A second precondition for innovation is the successful implementation. Well-established, old mechanisms usually introduce non-innovative reforms and changes. In public sector success is debatable because one group may be benefitted while, at the same time, disadvantage others. Success cannot be appraised properly. In the private sectors gains from innovation are translated into profits and market shares, thus payoffs can be evaluated.

\section{5. Factors and Pre-conditions for successful Innovation in education's management}

There is a variety of factors and pre-conditions that enhance the creation of innovative ideas and sustain the outcome in the management of education. The following list gives a representative illustration of these elements (Rivera León, Simmonds \& Roman 2012):

- Leadership is a vital factor of success. It includes the achievement of strategic alignments across an organization, the understanding of boundaries, the incentives to staff to take on actions and the collaboration across work units

- Culture, strategy and human capital. Innovation is more likely to happen in environments where a culture that encourages and rewards new ideas exists. A culture of trust, which gives authority to translating innovative ideas into practice, and embedding respect and good communication are thus essential. In addition, the work of top level managers is vital, in setting strategic directions to the organizations they lead.

- Understanding the environment. A good understanding of the focus of their organizations, the internal dynamics and its external environment is essential in order to meet the targets. The environment is volatile and changes constantly , requiring public organizations to be flexible enough to respond to these changes. Capturing evidence and having access to information through qualitative and quantitative data is vital in understanding the environment and reacting to it.

- Organizational capabilities and innovative capacity. Empowering and supporting staff responsible to bring innovative solutions into action, which in turn necessitates leadership, investment and commitment. Organizational flexibility and agility is needed in order to shift and obtain necessary skills and resources to meet emerging needs and opportunities. 
- Good governance. Governments should be accountable in respecting citizens freedoms, ensure the political stability and the absence of violence, be effective and provide quality services, regulate friendly policies and respect for the rule of law and control of corruption.

- sustained support of politicians, officials and suppliers. Political leaders and officials can establish a culture in which innovation is seen as natural. Organizations whose structures may change before the implementation of PSI, require projects that will embody low risk for a long term process.

\section{6. The application of technological innovation so to create efficiencies on education management}

Innovations applied to education into four modes: technological, processual, organizational and institutional (Kickert, 2005).

These innovations vary from new tools and methods to the formation of hierarchy in organizations and the transformation of state centrism institutions to network societies. Innovation models are complex procedures as a result of the number of participants and the activities involved. Early models were simplistic linear affairs and mainly about physical products and processes - the typical "technology push" or "demand pull" stereotypes. These have gradually evolved to more complex and interactive models, weaving different knowledge strands together. Such complex interactive models are particularly relevant in the context of services where users are a key part of the equation. Depending on the circumstances each model has a better application. It is not a case of one being better than the other but rather that we need different model for a different situation. In the following list we present a number of models for ways in which innovation can happen in education with the use of tecnology (Bessant \& Tidd, 2011):

- Research and Development led model

A concept is conceived by specialists, refined and launched. Investments in research and development lead to new products, services etc.

- High involvement innovation

This model stresses the ability of all employees to contribute to incremental problem solving innovation through what are often called continuous improvement' or 'kaizen' programs. Strategic objectives of an organization are clearly specified and understood. Targeted in this way high involvement innovation can deliver significant traction in areas like quality improvement, waste reduction and efficiency gains.

- Diffusion-centred

This model focuses on how to spread an idea rather than to generate a new idea. It is equally important to spread successfully and adopt an idea through a variety of participants as to create a new one. The main concern is to make the idea work.

\section{- Radical/discontinuous}

In this model a specialist works on a radical idea, being completely free, autonomy and break with conventional approaches. An early and famous example of this would be the 'skunk works' which Lockheed Martin set up to help them develop the for its time - impossible innovation of an invisible airplane. By allowing the group significant autonomy and keeping it separate from the mainstream it was possible to develop the stealth technologies which later became a mainstream innovation for the business. Public sector examples might include some of the radical policy think tanks and some of the Future Focus activity, but the question could also be raised about the relative absence of such models on the public sector innovation landscape.

\section{- Entrepreneur driven}

This model recognizes that much innovation arises from individual ideas in the early 'fluid' phase in the innovation life cycle. The value of an innovation model based on this is that it captures the fast creativity of diverse and enthusiastic individuals and small groups and may give important clues or even early entry to what becomes the dominant design for the future. It also underlines the venture capital model of growth, in which sponsors and entrepreneurs are connected to develop and scale innovations with a high level of novelty. This model has significance for the public sector since it potentially taps into the rich vein of social entrepreneurship distributed across individuals and groups around key regional, issues and concerns. 
It highlights the need for brokering and connecting to enable these entrepreneurs to flourish and their ideas to reach a wider audience - the amplifying effect. Examples might include The Hub, Innovation Exchange, BBC Backstage, Young Foundation, Education innovation challenge etc.

\section{- Recombinant innovation}

Innovation does not always involve pushing the frontiers of a particular market or technology; in some cases it can happen through transferring lessons from one world where they are well-developed into a new context. Key to making this happen is mechanisms to bridge across different worlds. Public sector examples might be the transferring of lean / six sigma principles which originated in manufacturing but could also include learning from radical experiments in different contexts - for example, Aravind eye clinics education institute and 'bottom of pyramid' (BoP) learning around health care, mobile banking and services in $\mathrm{BoP}$ markets.

\section{- User-led innovation}

Based on the pioneering work of Eric von Hippel work, this model recognises that users are often initiators or at least cocreators of innovation at the 'fuzzy front end'. Ideas may be developed into prototypes by user innovators and then be picked up on and produced/refined by professionals. Private sector interest in this approach has grown, not least as a consequence of the emergence of powerful selforganizing user communities - such as that surrounding Linux - which have become major sources of innovative ideas. There is now extensive use of 'crowd sourcing' and innovation competitions to mobilize expertise and insight at the front end of innovation.

In the public sector, in our case in educational management, there is considerable scope for this kind of activity - in Denmark it became the centrepiece of a major innovation initiative and led to the establishment of a specialist group - 24 Mindlab - with the mission of developing and diffusing user led approaches across the educational management. In the UK a variety of activities - such as the experience-based design work at educational institutes are examples of this approach.

\section{- Long term co-evolution}

This model relates to the specialized and occasional type of innovation in which transformational innovations emerge out of highly complex and chaotic environments. Under conditions where there are many different stakeholders and other elements - for example, technologies, markets, financial sources, etc. - it becomes impossible to predict the direction or long term trajectory of innovation. Instead complexity theory suggests something will eventually emerge as a product of 'co-evolution amongst these different interacting elements. An example might be the long-term picture of chronic disease management - we know that this is a growing problem involving a wide range of stakeholders - patients, health professionals, patient's associations, drug and medical companies, pension providers, etc. The growing incidence of chronic disease, its rising costs and increasing expectations mean that the current model is likely to be unable to deal with this challenge - but what replaces it is impossible to predict via simple extrapolation. Instead it will co-evolve out of the interactions of the various stakeholders. This does not lend itself to a structured innovation model but it is possible to develop some approaches to 'manage' innovation under these conditions. Complexity theory indicates that there are some patterns to complex system behaviour - for example, it is possible to identify 'attractor basins' zones where something begins to emerge - and to use amplifying feedback to enhance that to the point where it becomes a dominant design. The innovation management lessons here would be to be in 'there', engaged with the co-evolving space, be in there early, and to be in there actively, picking up on shifts which might become nodes around which radical new options emerge. A private sector equivalent might be the Danish diabetes care provider Novo Nordisk which invests $£ 1 \mathrm{~m} /$ year into the non-profit Oxford Health Alliance - a diverse group trying to work on chronic disease in radically different ways. This investment represents their 'lottery ticket' - by being close to the discussion they are 'in there' and 'in there early' and in a position to detect where early possible radical solutions might be going and to follow up on these. It is not clear where public sector equivalent organizations or approaches might be found though some of the think tanks might represent communities in which this might be happening. This was an indicative list as stated by iande.com, presenting relevant models operating in the public sector. Many combinations of the previous models can also happen. In addition, some models are better than others depending on the circumstance that are applied. More over in many cases an excellent solution is a combination of them.

\section{Discussion}


This paper presents an overview about innovations in the education management and how it can improve efficiencies. There is an extensive literature that covers the subject of innovation in the public sector, more precisely in the education management, sufficiently and detailed reports from institutional departments that document efforts globally. Still, innovation is an ongoing process as managers face volatile socio-political and economic environments. We made a comprehensive capture of published literature concerning technological innovations and we presented indicative cases where the efforts of managers are illustrated and assessed. Educational administration represent a significant part of the European socioeconomic activity. As the demand for educational services in many advanced countries is growing faster than the rest of the economy, it is essential for radicalinnovative solutions to be applied in order to address budget constraints and higher expectations of the users.

It must be said that educational management occurs through Networks, Market mechanisms and through methods that involve governments, schools, teachers, headmasters and the state bureaucracy. Innovation in education targets at the solution of major problems and the development of new opportunities. Innovation models are complex procedures as a result of the number of participants and the activities involved. Mainly they involve, research and development processes, synergies of public authorities with the private sector and focus on the diffusion process. All those lead into an efficient mode of management for school units.

\section{Conclusion}

Educational structure and practices are in the centre of great reforms. These reforms associated with New Public Management. NPM is a process that involves interaction between managers and markets. It is a set of cost-cutting and management concepts from the private sector including downsizing, entrepreneurialism, enterprise operations, quality management, customer service etc. According to that concept, school managers are trying to create a smaller, more responsive, more entrepreneurial and more effective public sector. Technological innovation has a key role on this and surely it can be the cornerstone of every change which may occur on this field of public administration.

Regarding the future of technological innovations in the management of educational institutes, organizational knowledge plays a significant role at launching and implementing innovative ideas. Knowledge management efforts typically focus on organizational objectives such as improved performance, competitive advantage etc. it is a changing mix of workers experience, values, expert insight, and intuition that provides an environmental framework for evaluating and incorporating new experiences and information.

\section{Suggestions for Further Research}

This paper tracks down the key issues on the Introduction and the application of technological innovations as administrative efficiency factor in education. The literature review indicated that there are many opportunities from the leverage of technological innovations. However, there is a need to proceed with a further research, which will be a qualitative research among educators so to examine this case.

\section{References}

[1] Ari-Veikko Anttiroiko, Stephen J. Bailey \& Pekka Valkama (2011). Innovations in Public Governance in the Western World. Innovation and the Public Sector, 15, 1-22.

[2] Buchanan, D.A. \& Huczynski, A. (2004). Organizational Behavior: An introductory text. Prentice Hall.

[3] Carlisle, H.M. (1982). Management: Concepts, Methods and Applications. Chicago: Science Research Associates Inc.

[4] Foot, M. \& Hook, C. (2008). Introducing Human Resource Management (5th ed.). Pearson Education Limited.

[5] Gerald E. Caiden \& Pushpinder S. Puniha (2011). Putting Public Governance Innovation into Perspective: From Administrative Reform to Innovation Discourse. Innovation and the Public Sector, 15, 23-38.

[6] Hersey, P. \& Blanchard, K.H (1969). Management of Organizational Behavior: Utilizing human Resources. Englewood cliffs, NJ: Prentice Hall. 
[7] Hugo Thenint, LL\&A (2010). Innovation in the public sector. Global Review of Innovation Intelligence and Policy Studies.

[8] John Bessant \& Joe Tidd (2011). Innovation and Entrepreneurship (2nd ed.). Wiley.

[9] Katsaros I. (2008). Organization and Administration of Education. Athens (In Greek).

[10] Lorena Rivera León, Paul Simmonds \& Laura Roman (2012). Trends and Challenges in Public Sector Innovation in Europe. Inno Policy Trend Chart.

[11] Mpourantas, D. (2005). Leadership: the road to lasting success (1st ed.). Athens.

[12] Naim Kapucu, (2010). New Public Management: Theory, Ideology, and Practice. Globalization: VIII Issues in Public Management,vol.40, pp.886-896.

[13] Pavlopoulos P. (1983). The administrative phenomenon under the theory of organizations. Athens-Komotini: A.N. Sakkoula publications.

[14] Saitis, Ch. (2008). Organization and Administration of Education (Self-Edition). Athens.

[15] Saitis, Ch. (1994). Basic issues of School management (Self-Edition). Athens.

[16] Saitis, Ch. (2000). Organization and Administration of Education. Athens.

[17] W.J.M. Kickert (2005). Public Governance: the Context of Administrative Innovations in Southern Europe. Organizing Innovation New Approaches to Cultural Change and Intervention in Public Sector Organizations, 5, 173-191).

[18] Zileznik, A. (2004). Managers and leaders: Are they different?. Harvard Business Review, 82(1), 74-81. 